\title{
The Existence of Fixed Point Theorems via $w$-Distance and $\alpha$-Admissible Mappings and Applications
}

\author{
Marwan Amin Kutbi ${ }^{1}$ and Wutiphol Sintunavarat ${ }^{2}$ \\ ${ }^{1}$ Department of Mathematics, King Abdulaziz University, P.O. Box 80203, Jeddah 21589, Saudi Arabia \\ ${ }^{2}$ Department of Mathematics and Statistics, Faculty of Science and Technology, Thammasat University, \\ Rangsit Center, Pathumthani 12121, Thailand
}

Correspondence should be addressed to Marwan Amin Kutbi; mkutbi@yahoo.com and Wutiphol Sintunavarat; poom_teun@hotmail.com

Received 23 June 2013; Revised 10 September 2013; Accepted 11 September 2013

Academic Editor: Wei-Shih Du

Copyright (c) 2013 M. A. Kutbi and W. Sintunavarat. This is an open access article distributed under the Creative Commons Attribution License, which permits unrestricted use, distribution, and reproduction in any medium, provided the original work is properly cited.

\begin{abstract}
We introduce the concept of the generalized $w_{\alpha}$-contraction mappings and establish the existence of fixed point theorem for such mappings by using the properties of $w$-distance and $\alpha$-admissible mappings. We also apply our result to coincidence point and common fixed point theorems in metric spaces. Further, the fixed point theorems endowed with an arbitrary binary relation are also derived from our results. Our results generalize the result of Kutbi, 2013, and several results in the literature.
\end{abstract}

\section{Introduction}

It is well known that many problems in many branches of mathematics can be transformed to a fixed point problem of the form $T x=x$ for self-mapping $T$ defined on framework of metric space $(X, d)$. In 1992, Banach [1] introduced the concept of contraction mapping and proved the fixed point theorem for such mapping, which is called the Banach contraction principle, which opened an avenue for further development of analysis in this field. Several mathematicians used different conditions on self-mappings and proved several fixed point theorems in metric spaces and other spaces.

In 1969, Nadler [2] established the fixed point theorem for multivalued contraction mapping by using the concept of Hausdorff metric which in turn is a generalization of the classical Banach contraction principle. Afterward, Kaneko [3] extended the corresponding results of Nadler [2] to single valued mapping and multivalued mapping which is also generalization of the result of Jungck [21]. Subsequently, there are a number of results that extend this result in many different directions (see in [4-13]).

On the other hand, Kada et al. [14] introduced the concept of $w$-distance on a metric space. Using this concept, they improved Caristi's fixed point theorem, Ekland's variational principle, and Takahashi's existence theorem. Afterward, Suzuki and Takahashi [15] established the fixed point result for multivalued mapping with respect to $w$-distance. In fact, this result is an improvement of the Nadler's fixed point theorem. Several fixed point theorems have been proved by many mathematicians in framework of metric spaces via $w$ distance; for example, see [16-19]. Recently, Kutbi [20] established useful lemma for $w$-distance which is an improved version of the lemma given in [21] and proved a key lemma on the existence of $f$-orbit for generalized $f$-contraction mappings. Also, he gave the existence of coincidence points and common fixed points for generalized $f$-contraction mappings not involving the extended Hausdorff metric.

The purpose of this work is to introduce the generalized $w_{\alpha}$-contraction mapping and prove fixed point theorem for such mapping via the concept of $\alpha$-admissible mapping of Mohammadi et al. [22], which is multivalued mapping version of $\alpha$-admissible mapping of Samet et al. [23] and different from the notion of $\alpha_{*}$-admissible which has been provided in [24] (also seen in [25-28]). The applications for coincidence point and common fixed point theorems in metric spaces and fixed point theorems endowed with an arbitrary binary 
relation are also derived from our results. Our results improve and complement the main result of Kutbi [20] and many results in the literature.

\section{Preliminaries}

In this section, we recall some definitions and lemmas of $w$-distance that will be required in the sequel. For metric space $(X, d)$, let $2^{X}, C l(X)$, and $C B(X)$ denote the collection of nonempty subsets of $X$, nonempty closed subsets of $X$, and nonempty closed bounded subsets of $X$, respectively. For $A, B \in C B(X)$, we define the Hausdorff distance with respect to $d$ by

$$
H(A, B)=\max \left\{\sup _{x \in A} d(x, B), \sup _{y \in B} d(y, A)\right\}
$$

for every $A, B \in C B(X)$, where $d(x, B)=\inf _{y \in B} d(x, y)$. It is well known that $(C B(X), H)$ is a metric space.

Definition 1. Let $(X, d)$ be a metric space, $f: X \rightarrow X$ a single valued mapping, and $T: X \rightarrow 2^{X}$ a multivalued mapping.

(1) A point $x \in X$ is called a fixed point of $T$ if $x \in T(x)$ and the set of fixed points of $T$ is denoted by $\mathscr{F}(T)$.

(2) A point $x \in X$ is called a coincidence point of $f$ and $T$ if $f(x) \in T(x)$. One denotes by $\mathscr{C}(f \cap T)$ the set of coincidence points of $f$ and $T$.

(3) A point $x \in X$ is called a common fixed point of $f$ and $T$ if $x=f(x) \in T(x)$. One denotes by $\mathscr{F}(f \cap T)$ the set of common fixed points of $f$ and $T$.

Definition 2. Let $(X, d)$ be a metric space, $f: X \rightarrow X$ a single valued mapping, and $T: X \rightarrow 2^{X}$ a multivalued mapping. The sequence $\left\{x_{n}\right\}$ in $X$ is said to be an $f$-orbit of $T$ at $x_{0} \in X$ if $f\left(x_{n}\right) \in T\left(x_{n-1}\right)$ for all $n \in \mathbb{N}$. In particular case, the sequence $\left\{x_{n}\right\}$ in $X$ is said to be an orbit of $T$ at $x_{0} \in X$ if $f$ is the identity mapping on $X$; that is, $x_{n} \in T\left(x_{n-1}\right)$ for all $n \in \mathbb{N}$.

Definition 3. Let $(X, d)$ a metric space, $f: X \rightarrow X$ a single valued mapping, and $T: X \rightarrow C B(X)$ a multivalued mapping.

(1) $T$ is said to be a contraction [2] if there exists a constant $\lambda \in(0,1)$ such that for each $x, y \in X$,

$$
H(T(x), T(y)) \leq \lambda d(x, y) .
$$

(2) $T$ is said to be an $f$-contraction [3] if there exists constant $\lambda \in(0,1)$, and for each $x, y \in X$,

$$
H(T(x), T(y)) \leq \lambda d(f(x), f(y)) .
$$

Definition 4 (see [14]). Let $(X, d)$ be a metric space. A function $\omega: X \times X \rightarrow[0, \infty)$ is called $w$-distance on $X$ if it satisfies the following for each $x, y, z \in X$ :

$$
\begin{aligned}
& \left(w_{1}\right) \omega(x, z) \leq \omega(x, y)+\omega(y, z) \\
& \left(w_{2}\right) \text { a mapping } \omega(x, \cdot): X \rightarrow[0, \infty) \text { is lower semicontin- } \\
& \quad \text { uous; }
\end{aligned}
$$

$\left(w_{3}\right)$ for any $\epsilon>0$, there exists $\delta>0$ such that $\omega(z, x) \leq \delta$ and $\omega(z, y) \leq \delta$ imply $d(x, y) \leq \epsilon$.

Let us give some examples of $w$-distance.

Example 5. Let $(X, d)$ be a metric space. Then, the metric $d$ is $w$-distance on $X$, but the converse is not true in general case. Therefore, the $w$-distance is a generalization of the metric.

Example 6. Let $(X, d)$ be a metric space. Then, a function $\omega$ : $X \times X \rightarrow[0, \infty)$ defined by $\omega(x, y)=c$ for all $x, y \in X$ is $w$-distance on $X$, where $c$ is a positive real number.

Example 7. Let $(X,\|\cdot\|)$ be a normed linear space. Then, a function $\omega: X \times X \rightarrow[0, \infty)$ defined by $\omega(x, y)=\|x\|+\|y\|$ for all $x, y \in X$ is $w$-distance on $X$.

Example 8. Let $(X,\|\cdot\|)$ be a normed linear space. Then, a function $\omega: X \times X \rightarrow[0, \infty)$ defined by $\omega(x, y)=\|y\|$ for all $x, y \in X$ is $w$-distance on $X$.

Remark 9. We obtain that in general for $x, y \in X$, $\omega(x, y) \neq \omega(y, x)$ and neither of the implications $\omega(x, y)=$ $0 \Leftrightarrow x=y$ necessarily holds.

Definition 10 (see [29]). Let $(X, d)$ be a metric space. One says that the $w$-distance $\omega$ on $X$ is a $w_{0}$-distance if $\omega(x, x)=0$ for all $x \in X$.

For more details of other examples and properties of the $w$-distance, one can refer to $[14,15,29]$. The following lemmas are useful for the main results in this paper.

Lemma 11 (see [14]). Let $(X, d)$ be a metric space and $\omega$ a $w$-distance on $X$. Suppose that $\left\{x_{n}\right\}$ and $\left\{y_{n}\right\}$ are sequences in $X$ and $\left\{\alpha_{n}\right\}$ and $\left\{\beta_{n}\right\}$ are sequences in $[0, \infty)$ converging to 0 . Then, the following hold for every $x, y, z \in X$ :

(1) if $\omega\left(x_{n}, y\right) \leq \alpha_{n}$ and $\omega\left(x_{n}, z\right) \leq \beta_{n}$ for any $n \in \mathbb{N}$, then $y=z$; in particular, if $\omega(x, y)=0$ and $\omega(x, z)=0$, then $y=z$;

(2) if $\omega\left(x_{n}, y_{n}\right) \leq \alpha_{n}$ and $\omega\left(x_{n}, z\right) \leq \beta_{n}$ for any $n \in \mathbb{N}$, then $\left\{y_{n}\right\}$ converges to $z$;

(3) if $\omega\left(x_{n}, x_{m}\right) \leq \alpha_{n}$ for any $n, m \in \mathbb{N}$ with $m>n$, then $\left\{x_{n}\right\}$ is a Cauchy sequence;

(4) if $\omega\left(y, x_{n}\right) \leq \alpha_{n}$ for any $n \in \mathbb{N}$, then $\left\{x_{n}\right\}$ is a Cauchy sequence.

Next, we give the definition of some type of mapping. Before giving next definition, we give the following notation. Let $(X, d)$ be a metric space and $\omega$ a $w$-distance on $X$. For $x \in X$ and $A \in 2^{X}$, we denote $\omega(x, A):=\inf _{y \in A} \omega(x, y)$.

Definition 12. Let $(X, d)$ be a metric space, $f: X \rightarrow X$ a singlevalued mapping, and $T: X \rightarrow C l(X)$ a multivalued mapping.

(1) $T$ is a $w$-contraction [15] if there exist a $w$-distance $\omega$ on $X$ and $\lambda \in(0,1)$ such that, for any $x, y \in X$ and $u \in T(x)$, there is $v \in T(y)$ with

$$
\omega(u, v) \leq \lambda \omega(x, y) \text {. }
$$


(2) $T$ is a generalized $f$-contraction [20] if there exist a $w_{0}$-distance $\omega$ on $X$ and $\lambda \in(0,1)$ such that, for any $x, y \in X$ and $u \in T(x)$, there is $v \in T(y)$ with

$$
\begin{aligned}
& \omega(u, v) \leq \lambda \max \{\omega(f(x), f(y)), \\
& \omega(f(x), T(x)), \omega(f(y), T(y)), \\
&\left.\frac{1}{2}[\omega(f(x), T(y))+\omega(f(y), T(x))]\right\} .
\end{aligned}
$$

Definition 13 (see $[22,24]$ ). Let $X$ be a nonempty set, $T: X \rightarrow$ $2^{X}$, where $2^{X}$ is a collection of subset of $X$ and $\alpha: X \times X \rightarrow$ $[0, \infty)$. One says that

(1) $T$ is an $\alpha_{*}$-admissible if, for all $x, y \in X$, one has

$$
\alpha(x, y) \geq 1 \Longrightarrow \alpha_{*}(T(x), T(y)) \geq 1
$$

where $\alpha_{*}(T(x), T(y)):=\inf \{\alpha(a, b): a \in T(x), b \in$ $T(y)\}$

(2) $T$ is an $\alpha$-admissible whenever, for each $x \in X$ and $y \in T x$ with $\alpha(x, y) \geq 1$, one has $\alpha(y, z) \geq 1$ for all $z \in T y$.

Remark 14. It is easy to see that $T$ is an $\alpha_{*}$-admissible mapping which implies $T$ as an $\alpha$-admissible mapping.

\section{Fixed Point Results}

In this section, we introduce the new mapping, the so-called generalized $w_{\alpha}$-contraction mapping, and prove the fixed point results for this mapping by using $w$-distance.

Definition 15. Let $(X, d)$ be a metric space and $\alpha: X \times X \rightarrow$ $[0, \infty)$ a given mapping. The multivalued mapping $T: X \rightarrow$ $C l(X)$ is said to be a generalized $w_{\alpha}$-contraction if there exist a $w_{0}$-distance $\omega$ on $X$ and $\lambda \in(0,1)$ such that, for any $x, y \in X$ and $u \in T(x)$, there is $v \in T(y)$ with

$$
\begin{array}{r}
\alpha(u, v) \omega(u, v) \\
\leq \lambda \max \{\omega(x, y), \omega(x, T(x)), \omega(y, T(y)), \\
\left.\frac{1}{2}[\omega(x, T(y))+\omega(y, T(x))]\right\} .
\end{array}
$$

Theorem 16. Let $(X, d)$ be a complete metric space, $\alpha: X \times$ $X \rightarrow[0, \infty)$, and $T: X \rightarrow C l(X)$ a generalized $w_{\alpha^{-}}$ contraction mapping. Suppose that the following conditions hold:

(a) $T$ is an $\alpha$-admissible mapping;

(b) there exist $x_{0} \in X$ and $x_{1} \in T\left(x_{0}\right)$ such that $\alpha\left(x_{0}, x_{1}\right) \geq 1$

(c) if for every $y \in X$ with $y \notin T(y)$, one has

$$
\inf \{\omega(x, y)+\omega(x, T(x)): x \in X\}>0,
$$

then, $\mathscr{F}(T) \neq \emptyset$.
Proof. For $x_{0} \in X$ and $x_{1} \in T\left(x_{0}\right)$ in (b), by the definition of generalized $w_{\alpha}$-contraction of $T$, there exists $x_{2} \in T\left(x_{1}\right)$ such that

$$
\begin{gathered}
\alpha\left(x_{1}, x_{2}\right) \omega\left(x_{1}, x_{2}\right) \\
\leq \lambda \max \left\{\omega\left(x_{0}, x_{1}\right), \omega\left(x_{0}, T\left(x_{0}\right)\right), \omega\left(x_{1}, T\left(x_{1}\right)\right),\right. \\
\left.\frac{1}{2}\left[\omega\left(x_{0}, T\left(x_{1}\right)\right)+\omega\left(x_{1}, T\left(x_{0}\right)\right)\right]\right\} .
\end{gathered}
$$

Since $x_{1} \in T\left(x_{0}\right), \alpha\left(x_{0}, x_{1}\right) \geq 1$, and $T$ is $\alpha$-admissible mapping, we have

$$
\alpha\left(x_{1}, x_{2}\right) \geq 1
$$

From (9) and (10), we have

$$
\begin{aligned}
& \omega\left(x_{1}, x_{2}\right) \\
& \leq \alpha\left(x_{1}, x_{2}\right) \omega\left(x_{1}, x_{2}\right) \\
& \leq \lambda \max \left\{\omega\left(x_{0}, x_{1}\right), \omega\left(x_{0}, T\left(x_{0}\right)\right), \omega\left(x_{1}, T\left(x_{1}\right)\right),\right. \\
& \left.\frac{1}{2}\left[\omega\left(x_{0}, T\left(x_{1}\right)\right)+\omega\left(x_{1}, T\left(x_{0}\right)\right)\right]\right\} .
\end{aligned}
$$

Similarly, using the definition of generalized $w_{\alpha}$-contraction of $T$, there exists $x_{3} \in T\left(x_{2}\right)$ such that

$$
\begin{gathered}
\alpha\left(x_{2}, x_{3}\right) \omega\left(x_{2}, x_{3}\right) \\
\leq \lambda \max \left\{\omega\left(x_{1}, x_{2}\right), \omega\left(x_{1}, T\left(x_{1}\right)\right), \omega\left(x_{2}, T\left(x_{2}\right)\right),\right. \\
\left.\frac{1}{2}\left[\omega\left(x_{1}, T\left(x_{2}\right)\right)+\omega\left(x_{2}, T\left(x_{1}\right)\right)\right]\right\} .
\end{gathered}
$$

From $x_{2} \in T\left(x_{1}\right), \alpha\left(x_{1}, x_{2}\right) \geq 1$, and $T$ is $\alpha$-admissible mapping; we have

$$
\alpha\left(x_{2}, x_{3}\right) \geq 1 \text {. }
$$

From (12) and (13), we have

$$
\begin{aligned}
& \omega\left(x_{2}, x_{3}\right) \leq \alpha\left(x_{2}, x_{3}\right) \omega\left(x_{2}, x_{3}\right) \\
& \leq \lambda \max \left\{\omega\left(x_{1}, x_{2}\right), \omega\left(x_{1}, T\left(x_{1}\right)\right),\right. \\
& \omega\left(x_{2}, T\left(x_{2}\right)\right), \\
&\left.\frac{1}{2}\left[\omega\left(x_{1}, T\left(x_{2}\right)\right)+\omega\left(x_{2}, T\left(x_{1}\right)\right)\right]\right\} .
\end{aligned}
$$


Continuing this process, we can construct the sequence $\left\{x_{n}\right\}$ in $X$ such that $x_{n} \in T\left(x_{n-1}\right)$,

$$
\alpha\left(x_{n}, x_{n+1}\right) \geq 1,
$$

$$
\begin{aligned}
\omega\left(x_{n}, x_{n+1}\right) & \\
\leq \lambda \max \{ & \omega\left(x_{n-1}, x_{n-2}\right), \omega\left(x_{n-1}, T\left(x_{n-1}\right)\right), \\
& \omega\left(x_{n-2}, T\left(x_{n-2}\right)\right), \\
& \left.\frac{1}{2}\left[\omega\left(x_{n-1}, T\left(x_{n-2}\right)\right)+\omega\left(x_{n-2}, T\left(x_{n-1}\right)\right)\right]\right\}
\end{aligned}
$$

for all $n \in \mathbb{N}$. Therefore, for each $n \in \mathbb{N}$, we have

$$
\begin{aligned}
& \omega\left(x_{n}, x_{n+1}\right) \\
& \leq \lambda \max \left\{\omega\left(x_{n-1}, x_{n}\right), \omega\left(x_{n-1}, T\left(x_{n-1}\right)\right), \omega\left(x_{n}, T\left(x_{n}\right)\right),\right. \\
& \left.\quad \frac{1}{2}\left[\omega\left(x_{n-1}, T\left(x_{n}\right)\right)+\omega\left(x_{n}, T\left(x_{n-1}\right)\right)\right]\right\} \\
& \leq \lambda \max \left\{\omega\left(x_{n-1}, x_{n}\right), \omega\left(x_{n-1}, x_{n}\right), \omega\left(x_{n}, x_{n+1}\right),\right. \\
& \left.\quad \frac{1}{2}\left[\omega\left(x_{n-1}, x_{n+1}\right)+\omega\left(x_{n}, x_{n}\right)\right]\right\} \\
& =\lambda \max \left\{\omega\left(x_{n-1}, x_{n}\right), \omega\left(x_{n}, x_{n+1}\right), \frac{1}{2}\left[\omega\left(x_{n-1}, x_{n+1}\right)\right]\right\} \\
& \leq \lambda \max \left\{\omega\left(x_{n-1}, x_{n}\right), \omega\left(x_{n}, x_{n+1}\right),\right. \\
& \left.\quad \frac{1}{2}\left[\omega\left(x_{n-1}, x_{n}\right)+\omega\left(x_{n}, x_{n+1}\right)\right]\right\}
\end{aligned}
$$$$
\leq \lambda \max \left\{\omega\left(x_{n-1}, x_{n}\right), \omega\left(x_{n}, x_{n+1}\right)\right\} \text {. }
$$

If there exists $n_{*} \in \mathbb{N}$ such that $\max \left\{\omega\left(x_{n_{*}-1}, x_{n_{*}}\right)\right.$, $\left.\omega\left(x_{n_{*}}, x_{n_{*}+1}\right)\right\}=\omega\left(x_{n_{*}}, x_{n_{*}+1}\right)$, then we have $\omega\left(x_{n_{*}}, x_{n_{*}+1}\right)=$ 0 , and then, $\omega\left(x_{n_{*}-1}, x_{n_{*}}\right)=0$. By property of $w$-distance, we get

$$
\omega\left(x_{n_{*}-1}, x_{n_{*}+1}\right) \leq \omega\left(x_{n_{*}-1}, x_{n_{*}}\right)+\omega\left(x_{n_{*}}, x_{n_{*}+1}\right)=0 .
$$

Since $\omega\left(x_{n_{*}-1}, x_{n_{*}}\right)=0$ and $\omega\left(x_{n_{*}-1}, x_{n_{*}+1}\right)=0$, using Lemma 11, we get $x_{n_{*}}=x_{n_{*}+1}$, and thus, $x_{n_{*}} \in T\left(x_{n_{*}}\right)$. This implies that $x_{n_{*}}$ is fixed point of $T$. Therefore, we may assume that $\max \left\{\omega\left(x_{n-1}, x_{n}\right), \omega\left(x_{n}, x_{n+1}\right)\right\}=\omega\left(x_{n-1}, x_{n}\right)$ for all $n \in \mathbb{N}$. From (17), we get

$$
\omega\left(x_{n}, x_{n+1}\right) \leq \lambda \omega\left(x_{n-1}, x_{n}\right)
$$

for all $n \in \mathbb{N}$. By Lemma 11, we have that $\left\{x_{n}\right\}$ converges in $X$.

By repeating (19), we obtain that

$$
\omega\left(x_{n}, x_{n+1}\right) \leq \lambda^{n} \omega\left(x_{0}, x_{1}\right)
$$

for all $n \in \mathbb{N}$.
For $m, n \in \mathbb{N}$ for which $m>n$, we get

$$
\begin{aligned}
\omega\left(x_{n}, x_{m}\right) \leq & \omega\left(x_{n}, x_{n+1}\right)+\omega\left(x_{n+1}, x_{n+2}\right) \\
& +\cdots+\omega\left(x_{m-1}, x_{m}\right) \\
\leq & \lambda^{n} \omega\left(x_{0}, x_{1}\right)+\lambda^{n+1} \omega\left(x_{0}, x_{1}\right) \\
& +\cdots+\lambda^{m-1} \omega\left(x_{0}, x_{1}\right) \\
\leq & \frac{\lambda^{n}}{1-\lambda} \omega\left(x_{0}, x_{1}\right) .
\end{aligned}
$$

Since $0<\lambda<1$, we get $\left(\lambda^{n} /(1-\lambda)\right) \omega\left(x_{0}, x_{1}\right) \rightarrow 0$ as $n \rightarrow \infty$. Using Lemma 11, we get $\left\{x_{n}\right\}$ as Cauchy sequence in $X$. By completeness of $X$, we get $x_{n} \rightarrow z$ as $n \rightarrow \infty$ for some $z \in X$. Since $\omega\left(x_{n}, \cdot\right)$ is lower semicontinuous, we have

$$
\begin{aligned}
\omega\left(x_{n}, z\right) & \leq \liminf _{m \rightarrow \infty} \omega\left(x_{n}, x_{m}\right) \\
& \leq \frac{\lambda^{n}}{1-\lambda} \omega\left(x_{0}, x_{1}\right) .
\end{aligned}
$$

Assuming that $z \notin T(z)$, then by hypothesis, we get

$$
\begin{aligned}
0 & <\inf \{\omega(x, z)+\omega(x, T(x)): x \in X\} \\
& \leq \inf \left\{\omega\left(x_{n}, z\right)+\omega\left(x_{n}, T\left(x_{n}\right)\right): n \in \mathbb{N}\right\} \\
& \leq \inf \left\{\omega\left(x_{n}, z\right)+\omega\left(x_{n}, x_{n+1}\right): n \in \mathbb{N}\right\} \\
& \leq \inf \left\{\frac{\lambda^{n}}{1-\lambda} \omega\left(x_{0}, x_{1}\right)+\lambda^{n} \omega\left(x_{0}, x_{1}\right): n \in \mathbb{N}\right\} \\
& =\left(\left\{\frac{2-\lambda}{1-\lambda}\right\} \omega\left(x_{0}, x_{1}\right)\right) \inf \left\{\lambda^{n}: n \in \mathbb{N}\right\} \\
& =0,
\end{aligned}
$$

which is contradicting. Therefore, $z \in T(z)$; that is, $z$ is fixed point of $T$. This completes the proof.

Corollary 17 (see Corollary 2.1 in [20]). Let $(X, d)$ be a complete metric space, let $\omega$ be $w_{0}$-distance on $X$, and let $T$ : $X \rightarrow \mathrm{Cl}(X)$ be a multivalued map satisfying the following:

(a) for each $x, y \in X$ and $u \in T(x)$, there exists $v \in T(y)$ such that

$$
\begin{gathered}
\omega(u, v) \leq \lambda \max \{\omega(x, y), \omega(x, T(x)), \omega(y, T(y)), \\
\left.\frac{1}{2}[\omega(x, T(y))+\omega(y, T(x))]\right\},
\end{gathered}
$$

where $\lambda \in(0,1)$;

(b) for every $y \in X$ with $y \notin T(y)$, one has

$$
\inf \{\omega(x, y)+\omega(x, T(x)): x \in X\}>0 .
$$

Then, $\mathscr{F}(T) \neq \emptyset$. 
Proof. Setting $\alpha(x, y)=1$ for all $x, y \in X$ in Theorem 16, we obtain the desired result.

Next, we give the notion of $w_{\alpha}$-contraction mapping and prove the existence of fixed point theorem for such mapping.

Definition 18. Let $(X, d)$ be a metric space and $\alpha: X \times X \rightarrow$ $[0, \infty)$. The multivalued mapping $T: X \rightarrow C l(X)$ is said to be $w_{\alpha}$-contraction if there exist a $w$-distance $\omega$ on $X$ and $\lambda \in(0,1)$ such that for any $x, y \in X$ and $u \in T(x)$, there is $v \in T(y)$ with

$$
\alpha(u, v) \omega(u, v) \leq \lambda \omega(x, y) .
$$

Theorem 19. Let $(X, d)$ be a complete metric space, $\alpha: X \times$ $X \rightarrow[0, \infty)$, and $T: X \rightarrow C l(X)$ a $w_{\alpha}$-contraction mapping. Suppose that the following conditions hold:

(a) $T$ is $\alpha$-admissible mapping;

(b) there exist $x_{0} \in X$ and $x_{1} \in T\left(x_{0}\right)$ such that $\alpha\left(x_{0}, x_{1}\right) \geq 1$

(c) for every $y \in X$ with $y \notin T(y)$, one has

$$
\inf \{\omega(x, y)+\omega(x, T(x)): x \in X\}>0 .
$$

Then, $\mathscr{F}(T) \neq \emptyset$.

Proof. We obtain that this result can be proven by using similar method in Theorem 16. Then, in order to avoid repetition, the details are omitted.

\section{Applications}

4.1. Application to Coincidence Point and Common Fixed Point Results. First of all, we introduce the following concept.

Definition 20. Let $X$ be a nonempty set, $f: X \rightarrow X, T$ : $X \rightarrow C l(X)$ such that $T(X) \subseteq f(X)$, where

$$
T(X):=\bigcup_{x \in X} T(x)
$$

and $\alpha: f(X) \times f(X) \rightarrow[0, \infty)$. One says that $T$ is $(f, \alpha)$ admissible whenever, for each $f(x) \in f(X)$ and $f(y) \in$ $T(f(x))$ with $\alpha(f(x), f(y)) \geq 1$, one has $\alpha(f(y), f(z)) \geq 1$ for all $f(z) \in T(f(y))$.

Remark 21. If $T$ is $(f, \alpha)$-admissible and $f$ is the identity mapping, then $T$ is $\alpha$-admissible.

Definition 22. Let $(X, d)$ be a metric space, $f: X \rightarrow X$, and $\alpha: f(X) \times f(X) \rightarrow[0, \infty)$. The multivalued mapping $T:$ $X \rightarrow C l(X)$ is said to be generalized $\left(w_{\alpha}, f\right)$-contraction if there exist a $w_{0}$-distance $\omega$ on $X$ and $\lambda \in(0,1)$ such that, for any $x, y \in X$ and $u \in T(x)$, there is $v \in T(y)$ with

$$
\begin{aligned}
& \alpha(f(x), f(y)) \omega(u, v) \\
& \leq \lambda \max \{\omega(f(x), f(y)), \omega(f(x), T(x)), \omega(f(y), T(y)), \\
& \left.\frac{1}{2}[\omega(f(x), T(y))+\omega(f(y), T(x))]\right\} .
\end{aligned}
$$

Remark 23. If $T$ is generalized $\left(w_{\alpha}, f\right)$-contraction and $f$ is the identity mapping, then $T$ is generalized $w_{\alpha}$-contraction.

Next, we give useful lemma of Haghi et al. [30].

Lemma 24 (see [30]). Let $X$ be a nonempty set and $f: X \rightarrow$ $X$ a mapping. Then, there exists a subset $E \subseteq X$ such that $f(E)=f(X)$ and $f: E \rightarrow X$ is one-to-one.

Now, we apply our result in Section 3 to the coincidence point theorem by using Lemma 24.

Theorem 25. Let $(X, d)$ be a complete metric space, $f: X \rightarrow$ $X, \alpha: f(X) \times f(X) \rightarrow[0, \infty)$, and $T: X \rightarrow C l(X)$ a generalized $\left(w_{\alpha}, f\right)$-contraction. Suppose that the following conditions hold:

(A) $T(X) \subseteq f(X)$;

(B) $T$ is $(f, \alpha)$-admissible;

(C) there exist $x_{0}, x_{1} \in X$ such that $f\left(x_{1}\right) \in T\left(x_{0}\right)$ and $\alpha\left(f\left(x_{0}\right), f\left(x_{1}\right)\right) \geq 1 ;$

(D) for all $y \in X$ with $f(y) \notin T(y)$, one has

$\inf \{\omega(f(x), f(y))+\omega(f(x), T(x)): x \in X\}>0$.

Then, $\mathscr{C}(f \cap T) \neq \emptyset$.

Proof. Consider the mapping $f: X \rightarrow X$. Using Lemma 24, there exists $E \subseteq X$ such that $f(E)=f(X)$ and $\left.f\right|_{E}$ is oneto-one. Now, we can define a mapping $\mathscr{H}: f(E) \rightarrow C l(X)$ by

$$
\mathscr{H}(f(x))=T(x)
$$

for all $x \in E$. Follows from $\left.f\right|_{E}$ is one-to-one that $\mathscr{H}$ is welldefined.

Since $T$ is a generalized $\left(w_{\alpha}, f\right)$-contraction, there exist a $w_{0}$-distance $\omega$ on $X$ and $\lambda \in(0,1)$ such that, for any $x, y \in X$ and $u \in T(x)$, there is $v \in T(y)$ with

$$
\begin{gathered}
\alpha(f(x), f(y)) \omega(u, v) \\
\leq \lambda \max \{\omega(f(x), f(y)), \omega(f(x), T(x)), \omega(f(y), T(y)), \\
\left.\frac{1}{2}[\omega(f(x), T(y))+\omega(f(y), T(x))]\right\} .
\end{gathered}
$$

By the construction of $\mathscr{H}$, for any $f(x), f(y) \in f(E)$, and $u \in \mathscr{H}(f(x))$, there is $v \in \mathscr{H}(f(y))$ such that

$$
\begin{aligned}
\alpha(f(x), f(y)) \omega(u, v) \\
\leq \lambda \max \{\omega(f(x), f(y)), \omega(f(x), \mathscr{H}(f(x))), \\
\omega(f(y), \mathscr{H}(f(y))), \\
\left.\frac{1}{2}[(f(x), \mathscr{H}(f(y)))+\omega(f(y), \mathscr{H}(f(x)))]\right\} .
\end{aligned}
$$


This implies that $\mathscr{H}$ is a generalized $w_{\alpha}$-contraction. Since $T$ is $(f, \alpha)$-admissible, we have $\mathscr{H}$ as $\alpha$-admissible. It is obtained that condition (C) implies condition (B) in Theorem 16. From (D), for all $f(y) \in f(E)$ with $f(y) \notin \mathscr{H}(f(y))$, we have

$$
\inf \{\omega(f(x), f(y))+\omega(f(x), \mathscr{H}(f(x))): x \in X\}>0 .
$$

Using Theorem 16 with mapping $\mathscr{H}$, we can find a fixed point of mapping $\mathscr{H}$.

Let $z$ be fixed point of $\mathscr{H}$; that is, $z \in \mathscr{H}(z)$. Since $z \in$ $f(E)$, we can find $\widehat{z} \in E$ such that $z=f(\widehat{z})$. Now, we have

$$
f(\widehat{z})=z \in \mathscr{H}(z)=\mathscr{H}(f(\widehat{z}))=T(\widehat{z}) .
$$

Therefore, $\widehat{z}$ is a coincident point of $f$ and $T$; that is, $\mathscr{C}(f \cap$ $T) \neq \emptyset$. This completes the proof.

Finally, we obtain a common fixed point result. Before giving our results, we need a few definitions.

Definition 26. Let $(X, d)$ be a metric space, $f: X \rightarrow X$, and $T: X \rightarrow C l(X)$. Mappings $f$ and $T$ are said to commute weakly if

$$
f(T(x)) \subseteq T(f(X))
$$

for all $x \in X$.

Theorem 27. Suppose that all the hypotheses of Theorem 25 hold. Further, if $f$ and $T$ commute weakly and satisfy the following condition for $x \in X$ :

$$
f(x) \neq f^{2}(x) \Longrightarrow f(x) \notin T(x),
$$

then, $\mathscr{F}(f \cap T) \neq \emptyset$.

Proof. From Theorem 25, $f$ and $T$ have a coincidence point $\widehat{z} \in X$; that is, $f(\widehat{z}) \in T(\widehat{z})$. By the hypothesis, we get $f(\widehat{z})=$ $f^{2}(\widehat{z})$. It follows from $f$ and $T$ which commute weakly that

$$
f(\widehat{z})=f(f(\widehat{z})) \in f(T(\widehat{z})) \subseteq T(f(\widehat{z})) .
$$

This implies that $f(\widehat{z})$ is a common fixed point of $f$ and $T$, and thus, $\mathscr{F}(f \cap T) \neq \emptyset$. This completes the proof.

Remark 28. If we set $\alpha(a, b)=1$ for all $a, b \in f(X)$ in Theorems 25 and 27, then we get Theorems 2.1 and 2.2 of Kutbi [20].

4.2. Application to Fixed Point on Metric Space Endowed with an Arbitrary Binary Relation. In this section, we give the existence of fixed point theorems on a metric space endowed with an arbitrary binary relation.

Before presenting our results, we need a few definitions. Let $(X, d)$ be a metric space and $\mathscr{R}$ a binary relation over $X$. Denote that

$$
\mathcal{S}:=\mathscr{R} \cup \mathscr{R}^{-1}
$$

this is the symmetric relation attached to $\mathscr{R}$. Clearly,

$$
x, y \in X, \quad x \mathcal{S} y \Longleftrightarrow x \mathscr{R} y \text { or } y \mathscr{R} x \text {. }
$$

Definition 29. Let $(X, d)$ be a metric space and $\mathscr{R}$ a binary relation over $X$. One says that $T: X \rightarrow C l(X)$ is a comparative mapping with respect to $\mathcal{S}$ if, for each $x \in X$ and $y \in T(x), x \mathcal{S} y$ implies $y \mathcal{S} z$ for all $z \in T(y)$.

Definition 30. Let $(X, d)$ be a metric space and $\mathscr{R}$ a binary relation over $X$. The multivalued mapping $T: X \rightarrow \mathrm{Cl}(X)$ is said to be a generalized $w$-contraction with respect to $\mathcal{S}$ if there exist a $w_{0}$-distance $\omega$ on $X$ and $\lambda \in(0,1)$ such that, for any $x, y \in X$ for $x \mathcal{S} y$ and $u \in T(x)$, there is $v \in T(y)$ with

$$
\begin{gathered}
\omega(u, v) \leq \lambda \max \{\omega(x, y), \omega(x, T(x)), \omega(y, T(y)), \\
\left.\frac{1}{2}[\omega(x, T(y))+\omega(y, T(x))]\right\} .
\end{gathered}
$$

Theorem 31. Let $(X, d)$ be a complete metric space, $\mathscr{R}$ a binary relation over $X$, and $T: X \rightarrow C l(X)$ a generalized $w$-contraction with respect to $\mathcal{S}$. Suppose that the following conditions hold:

(A) $T$ is a comparative mapping with respect to $\mathcal{S}$;

(B) there exist $x_{0} \in X$ and $x_{1} \in T\left(x_{0}\right)$ such that $x_{0} \delta x_{1}$;

(C) for all $y \in X$ with $y \notin T(y)$, one has

$$
\inf \{\omega(x, y)+\omega(x, T(x)): x \in X\}>0 .
$$

Then, $\mathscr{F}(T) \neq \emptyset$.

Proof. Consider the mapping $\alpha: X \times X \rightarrow[0, \infty)$ defined by

$$
\alpha(x, y)= \begin{cases}1, & x \mathcal{S} y \\ 0, & \text { otherwise }\end{cases}
$$

From condition (B), we get $\alpha\left(x_{0}, x_{1}\right) \geq 1$. It follows from $T$ as comparative mapping with respect to $\mathcal{S}$ that $T$ is $\alpha$-admissible mapping. Since $T$ is generalized $w$-contraction with respect to $\mathcal{S}$, for any $x, y \in X$ and $u \in T(x)$, there is $v \in T(y)$ with

$$
\begin{array}{r}
\alpha(x, y) \omega(u, v) \leq \lambda \max \{\omega(x, y), \omega(x, T(x)), \omega(y, T(y)), \\
\left.\frac{1}{2}[\omega(x, T(y))+\omega(y, T(x))]\right\} .
\end{array}
$$

This implies that $T$ is generalized $w_{\alpha}$-contraction mapping. Now, all the hypotheses of Theorem 16 are satisfied, and so the existence of the fixed point of $T$ follows from Theorem 16. Therefore, $\mathscr{F}(T) \neq \emptyset$.

Next, we deduce Theorem 31 to the special case in the context of partially ordered metric spaces. Before studying the next results, we give the following concepts.

Definition 32. Let $X$ be a nonempty set. Then, $(X, d, \preceq)$ is called a partially ordered metric space if $(X, d)$ is a metric space and $(X, \preceq)$ is a partially ordered set. 
For partially ordered metric space $(X, d, \preceq)$ and $x, y \in X$, we denote that

$$
\asymp:=\{(x, y) \in X \times X: x \preceq y \text { or } y \preceq x\} .
$$

Definition 33. Let $(X, d, \preceq)$ be a partially ordered metric space. One says that $T: X \rightarrow C l(X)$ is a comparative mapping with respect to $\approx$ if, for each $x \in X$ and $y \in T(x)$, $x=y$ implies $y=z$ for all $z \in T(y)$.

Definition 34 . Let $(X, d, \preceq)$ be a partially ordered metric space. The multivalued mapping $T: X \rightarrow C l(X)$ is said to be a generalized $w$-contraction with respect to $\asymp$ if there exist a $w_{0}$-distance $\omega$ on $X$ and $\lambda \in(0,1)$ such that, for any $x, y \in X$ for which $x=y$ and $u \in T(x)$, there is $v \in T(y)$ with

$$
\begin{gathered}
\omega(u, v) \leq \lambda \max \{\omega(x, y), \omega(x, T(x)), \omega(y, T(y)), \\
\left.\frac{1}{2}[\omega(x, T(y))+\omega(y, T(x))]\right\} .
\end{gathered}
$$

Corollary 35. Let $(X, d, \preceq)$ be a partially ordered metric space and $T: X \rightarrow C l(X)$ a generalized $w$-contraction with respect to $\asymp$. Suppose that the following conditions hold:

(A) $T$ is a comparative mapping with respect to $\asymp$;

(B) there exist $x_{0} \in X$ and $x_{1} \in T\left(x_{0}\right)$ such that $x_{0}=x_{1}$;

(C) for all $y \in X$ with $y \notin T(y)$,

$$
\inf \{\omega(x, y)+\omega(x, T(x)): x \in X\}>0 .
$$

Then, $\mathscr{F}(T) \neq \emptyset$.

Proof. Since $\preceq$ is a binary operation on $X$, this result follows from Theorem 31 .

\section{Acknowledgment}

The first author gratefully acknowledges the support from the Deanship of Scientific Research (DSR) at King Abdulaziz University (KAU) during this research.

\section{References}

[1] S. Banach, "Sur les opérations dans les ensembles abstraits et leur applications aux équations intégrales," Fundamenta Mathematicae, vol. 3, pp. 133-181, 1922.

[2] S. B. Nadler, "Multi-valued contraction mappings," Pacific Journal of Mathematics, vol. 30, pp. 475-488, 1969.

[3] H. Kaneko, "Single-valued and multivalued $f$-contractions," Bollettino dell'Unione Matematica Italiana, vol. 6, no. 4, pp. 2933, 1985.

[4] N. Hussain and A. Alotaibi, "Coupled coincidences for multivalued nonlinear contractions in partially ordered metric spaces," Fixed Point Theory and Applications, vol. 2011, p. 81, 2011.

[5] P. Z. Daffer and H. Kaneko, "Multi-valued $f$-contractive mappings," Bollettino dell'Unione Matematica Italiana A, vol. 8, no. 7, pp. 233-241, 1994.

[6] H. Kaneko and S. Sessa, "Fixed point theorems for compatible multi-valued and single-valued mappings," International Journal of Mathematics and Mathematical Sciences, vol. 12, no. 2, pp. 257-262, 1989.
[7] A. Latif and I. Tweddle, "Some results on coincidence points," Bulletin of the Australian Mathematical Society, vol. 59, no. 1, pp. 111-117, 1999.

[8] H. K. Pathak, "Fixed point theorems for weak compatible multi-valued and single-valued mappings," Acta Mathematica Hungarica, vol. 67, no. 1-2, pp. 69-78, 1995.

[9] H. K. Pathak and M. S. Khan, "Fixed and coincidence points of hybrid mappings," Archivum Mathematicum, vol. 38, no. 3, pp. 201-208, 2002.

[10] W. Sintunavarat and P. Kumam, "Coincidence and common fixed points for hybrid strict contractions without the weakly commuting condition," Applied Mathematics Letters, vol. 22, no. 12, pp. 1877-1881, 2009.

[11] W. Sintunavarat and P. Kumam, "Weak condition for generalized multi-valued $(f, \alpha, \beta)$-weak contraction mappings," Applied Mathematics Letters, vol. 24, no. 4, pp. 460-465, 2011.

[12] W. Sintunavarat and P. Kumam, "Common fixed point theorem for hybrid generalized multi-valued contraction mappings," Applied Mathematics Letters, vol. 25, no. 1, pp. 52-57, 2012.

[13] W. Sintunavarat and P. Kumam, "Common fixed point theorem for cyclic generalized multi-valued contraction mappings," Applied Mathematics Letters, vol. 25, no. 11, pp. 1849-1855, 2012.

[14] O. Kada, T. Suzuki, and W. Takahashi, "Nonconvex minimization theorems and fixed point theorems in complete metric spaces," Mathematica Japonica, vol. 44, no. 2, pp. 381-391, 1996.

[15] T. Suzuki and W. Takahashi, "Fixed point theorems and characterizations of metric completeness," Topological Methods in Nonlinear Analysis, vol. 8, no. 2, pp. 371-382, 1996.

[16] B. A. Bin Dehaish and A. Latif, "Fixed point results for multivalued contractive maps," Fixed Point Theory and Applications, vol. 2012, article 61, 2012.

[17] A. Latif and A. A. N. Abdou, "Fixed points of generalized contractive maps," Fixed Point Theory and Applications, vol. 2009, Article ID 487161, 9 pages, 2009.

[18] A. Latif and A. A. N. Abdou, "Multivalued generalized nonlinear contractive maps and fixed points," Nonlinear Analysis. Theory, Methods \& Applications, vol. 74, no. 4, pp. 1436-1444, 2011.

[19] T. Suzuki, "Generalized distance and existence theorems in complete metric spaces," Journal of Mathematical Analysis and Applications, vol. 253, no. 2, pp. 440-458, 2001.

[20] M. A. Kutbi, " $f$-contractive multivalued maps and coincidence points," Journal of Inequalities and Applications, vol. 2013, article 141, 2013.

[21] G. Jungck, "Commuting mappings and fixed points," The American Mathematical Monthly, vol. 83, no. 4, pp. 261-263, 1976.

[22] B. Mohammadi, S. Rezapour, and N. Shahzad, "Some results on fixed points of $\alpha-\psi$-ciric generalized multifunctions," Fixed Point Theory and Applications, vol. 2013, article 24, 2013.

[23] B. Samet, C. Vetro, and P. Vetro, "Fixed point theorems for $\alpha-\psi$-contractive type mappings," Nonlinear Analysis: Theory, Methods \& Applications, vol. 75, no. 4, pp. 2154-2165, 2012.

[24] J. H. Asl, S. Rezapour, and N. Shahzad, "On fixed points of $\alpha-\psi$-contractive multifunctions," Fixed Point Theory and Applications, vol. 2012, article 212, 2012.

[25] R. P. Agarwal, W. Sintunavarat, and P. Kumam, "PPF dependent fixed point theorems for an $\alpha_{c}$-admissible non-self mapping in the Razumikhinclass," Fixed Point Theory and Applications. In press.

[26] N. Hussain, P. Salimi, and A. Latif, "Fixed point results for single and set-valued $\alpha-\eta-\psi$-contractive mappings," Fixed Point Theory and Applications, vol. 2013, article 212, 2013. 
[27] N. Hussain, M. A. Kutbi, and P. Salimi, "Best proximity point results for modified $\alpha-\psi$-proximal rational contractions," Abstract and Applied Analysis, vol. 2013, Article ID 927457, 14 pages, 2013.

[28] P. Salimi, A. Latif, and N. Hussain, "Modified $\alpha-\psi$-contractive mappings with applications," Fixed Point Theory and Applications, vol. 2013, article 151, 2013.

[29] W.-S. Du, "Fixed point theorems for generalized Hausdorff metrics," International Mathematical Forum, vol. 3, no. 21-24, pp. 1011-1022, 2008.

[30] R. H. Haghi, Sh. Rezapour, and N. Shahzad, "Some fixed point generalizations are not real generalizations," Nonlinear Analysis: Theory, Methods \& Applications, vol. 74, no. 5, pp. 1799-1803, 2011. 


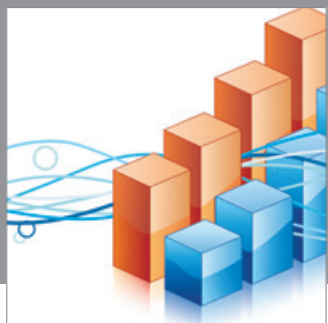

Advances in

Operations Research

mansans

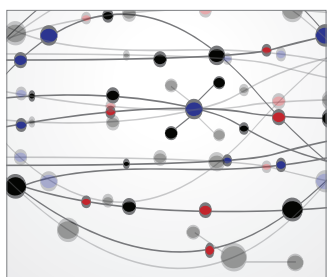

The Scientific World Journal
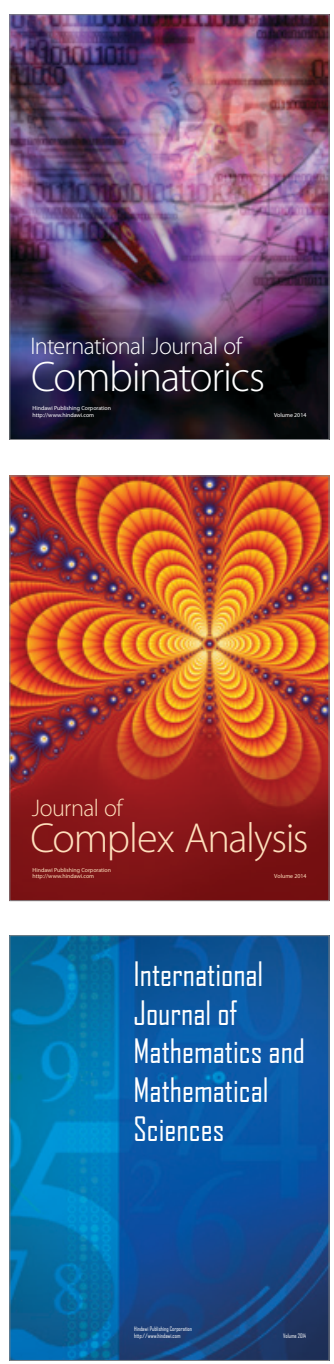
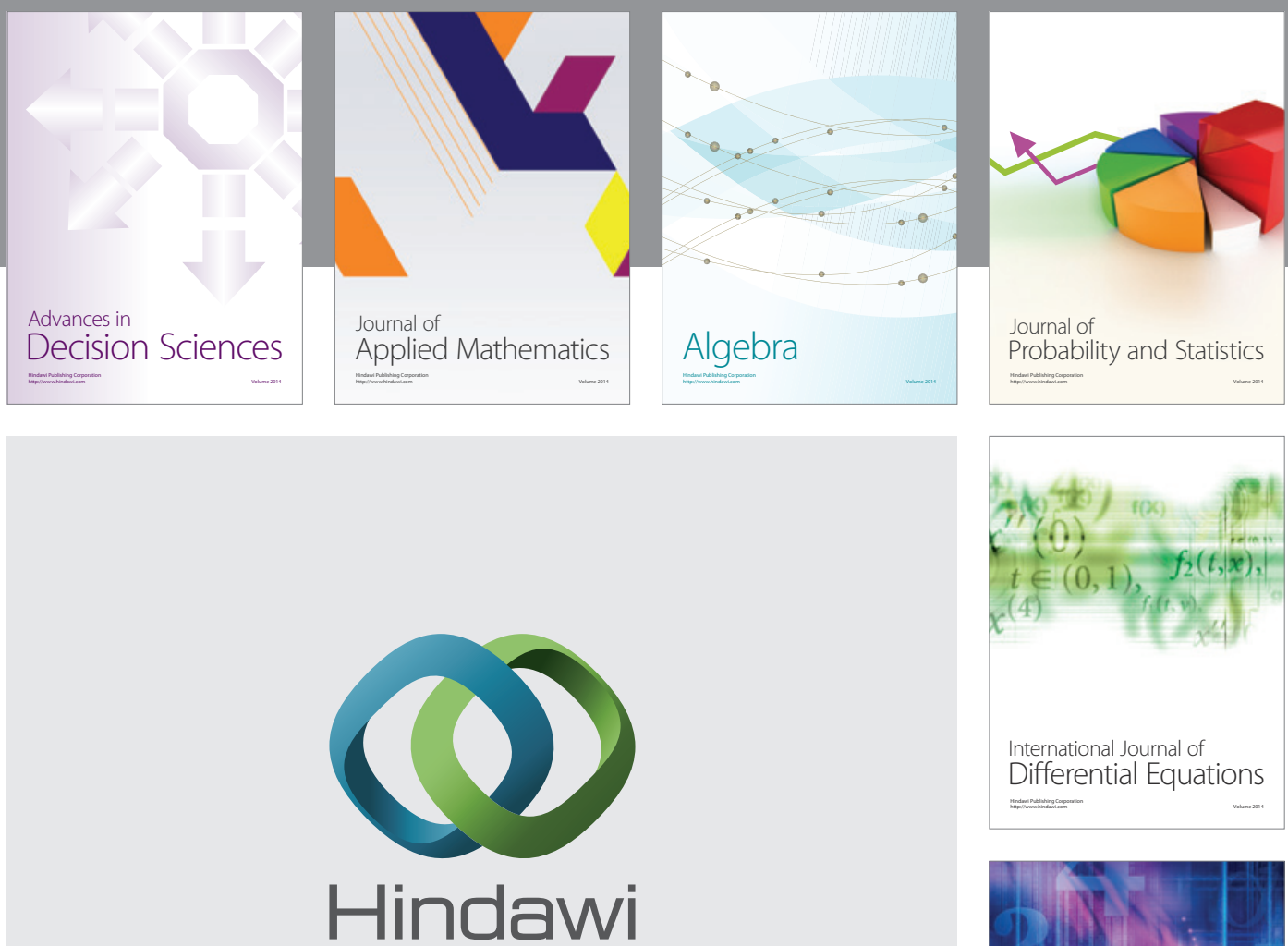

Submit your manuscripts at http://www.hindawi.com
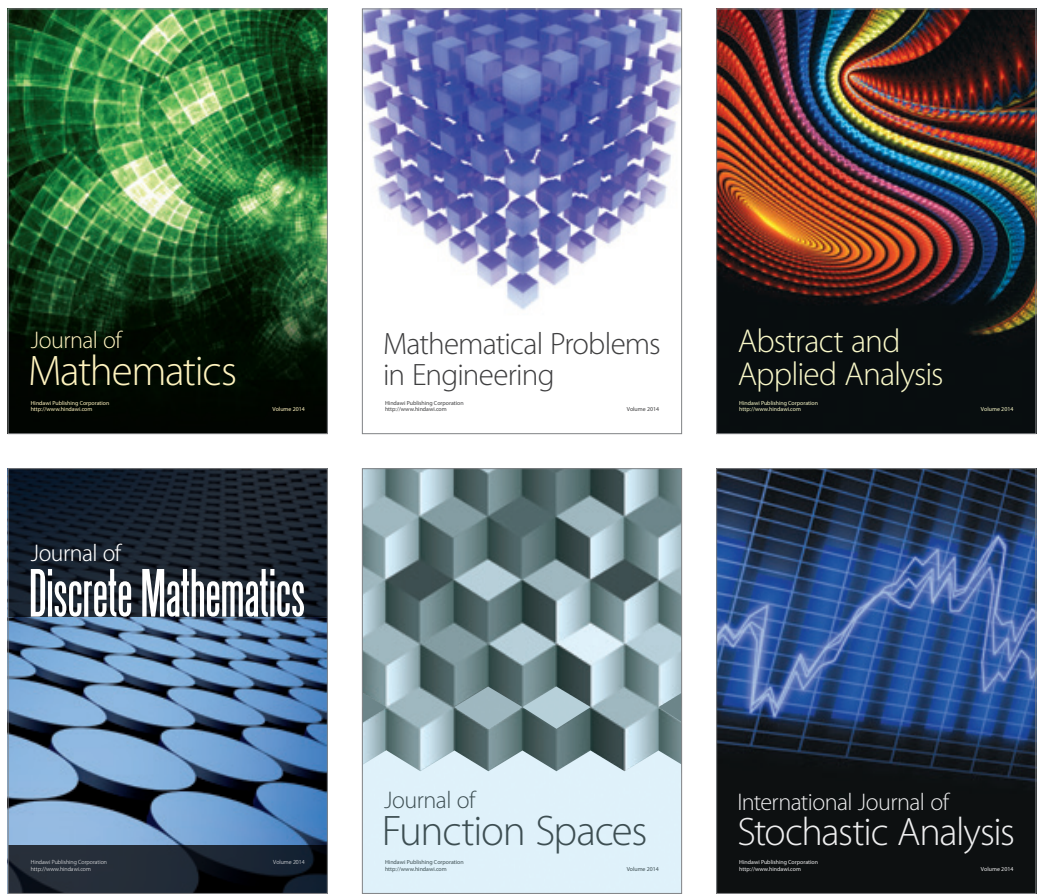

Journal of

Function Spaces

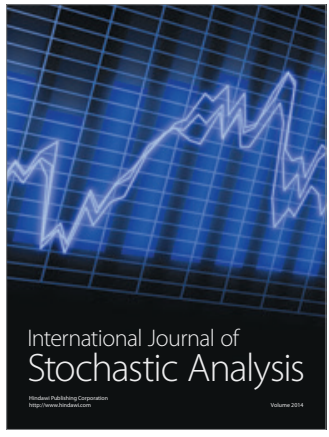

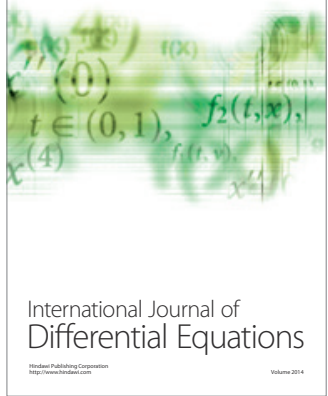
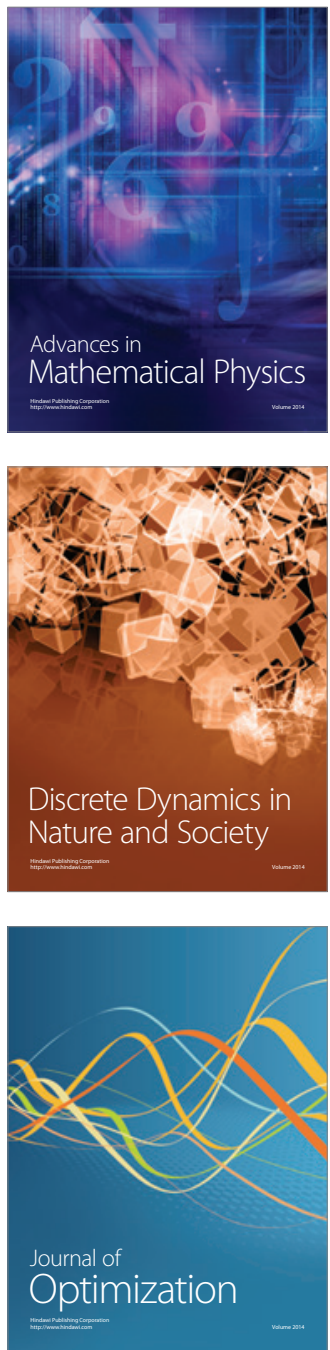